\title{
Ultrasound surveillance on detection of nonpalpable supraclavicular recurrence after breast cancer surgery
}

\author{
Eun Cho ${ }^{1}$, Jin Hwa Lee ${ }^{1}$, Eun Hwa Park ${ }^{2}$, Kyung Do Byun ${ }^{2}$ \\ ${ }^{1}$ Department of Radiology, ${ }^{2}$ Department of Surgery, Dong-A University Hospital, Busan, South Korea
}

\begin{abstract}
Aims: To evaluate the clinical utility of supraclavicular scanning for locoregional lymph node (LN) assessment in postoperative screening surveillance using ultrasonography (US) in patients who underwent breast cancer surgery. Material and methods: From July 2004 to February 2019, 280 suspicious findings for locoregional recurrence in the lymph node (LRL) on postoperative screening US were detected in 266 asymptomatic patients who underwent breast cancer surgery. Suspicious features of LRL on US included the marked hypoechogenicity, round or irregular shape, eccentric cortical thickening and replacement of the fatty hilum of the LNs. The bilateral breasts, including mastectomy sites, bilateral axillae, internal mammary areas and supraclavicular areas, were included in the scan range of postoperative US. Results: Of 280 LNs with suspicious findings for LRL, LRL of supraclavicular LNs was confirmed in 24 LNs according to cytopathology results. Multivariate logistic regression analysis revealed that higher overall staging of primary breast cancer (odds ratio [OR] 2.361 [95\% confidence interval (CI) 1.302-4.282]; $\mathrm{p}=0.005)$, higher $\mathrm{N}$ staging of primary breast cancer (OR 3.086 [95\% CI 1.479-6.441]; $\mathrm{p}=0.003$ ), older patient age (OR 1.060 [95\% CI 1.026-1.095]; $\mathrm{p}<0.001$ ) and breast-conserving surgery (OR 2.253 [95\% CI 1.184-4.289]; $\mathrm{p}=0.013$ ) were independently associated with LRL of supraclavicular LNs. Tumor size, nuclear grade, histological type, hormonal receptor status of the primary cancer, and bilateral cancer were not associated with $\operatorname{LRL}(\mathrm{p}=0.216, \mathrm{p}=0.205, \mathrm{p}=0.789$, $\mathrm{p}=0.899$, and $\mathrm{p}=0.900$, respectively). Conclusion: Routine supraclavicular scanning in postoperative screening surveillance using US in breast cancer patients with higher staging could be useful for the detection of LRL of supraclavicular LNs.
\end{abstract}

Keywords: ultrasonography; lymph nodes; supraclavicular; breast neoplasms; recurrence

\section{Introduction}

Breast cancer is the most common cancer and also one of the leading causes of death among women in developing countries $[1,2]$. Over recent decades, the early detection of and treatment strategies for breast cancer have changed tremendously. As a result, the survival of patients with the breast cancer has been improved significantly $[3,4]$. However, women with breast cancer who

Received 23.11.2019 Accepted 01.03.2020

Med Ultrason

2020, Vol. 22, No 2, 171-177

Corresponding author: Jin Hwa Lee, MD, PhD

49201, Busan, South Korea

Phone: $(+82) 512405367$

Fax: (+82) 512534931

Email: jhrad@dau.ac.kr experience locoregional recurrence after surgery have an increased risk for distant metastasis and poor prognosis, which remains a major problem for postoperative management of those with breast cancer [5-10].

According to the American College of Radiology (ACR) guidelines, ultrasonography (US) is not the recommended modality for postoperative screening in patients with a personal history of breast cancer $[11,12]$. The false-positive detection rate of locoregional recurrence in lymph nodes (LRL) in postoperative screening surveillance using US has been noted to be high and US is reported as inefficient for evaluating the supraclavicular area due to its time-consuming posture changes and low incidence of supraclavicular LRL $[13,14]$. Nevertheless, it has been also reported that, postoperative screening surveillance using US has been useful for the early detection of LRL and for diagnosis of postopera- 
tive complications because its scan range can cover the breasts, mastectomy site and locoregional lymph nodes (LNs) [13,15-18]. LRL after mastectomy is relatively uncommon compared with recurrence in the chest wall, but it has been associated with higher rates of simultaneous distant metastases and poor disease-specific survival [19-23]. Among the LRL, isolated supraclavicular LRL could be a curable condition and it has been reported that the early detection of LRL could be helpful in improving prognosis in patients after surgery [13-15,24,25].

Therefore, the present study aimed to evaluate the clinical utility of routine supraclavicular scanning for locoregional LNs assessment in postoperative screening surveillance using US in patients who underwent breast cancer surgery. Furthermore, we also aimed to identify patients who require routine supraclavicular US scanning based on analysis of clinicopathological characteristics of those with supraclavicular LRL detected using US.

\section{Material and methods}

Due to the retrospective nature of the present study and the use of anonymized patient data, requirement for informed consent was waived. However, informed consent was obtained for US-guided fine-needle aspiration biopsy (US-FNAB) and surgery from all patients before each procedure. The present study was approved by the institutional review board.

\section{Patients}

Between July 2004 and February 2019, 20,716 postoperative US screening surveillance examinations were performed in 2,144 women at the authors' institution. Among these 2,144 patients, we included 266 patients that underwent breast cancer surgery, with 280 suspicious findings for LRL on postoperative screening surveillance US in this study. We excluded the patients with suspicious LRL findings found only on computed tomography (CT) or magnetic resonance imaging (MRI) without undergoing postoperative screening surveillance with US, those with local recurrence and with palpable LNs.

A total of 256 patients had suspicious findings for LRL only in one of the axillae, the internal mammary area and the supraclavicular area, 4 patients exhibited suspicious findings in both axillae, the internal mammary area and the supraclavicular area, 4 patients had suspicious findings in both the internal mammary area and the supraclavicular area and 2 patients had suspicious findings for LRL in both axillae and the supraclavicular area. For LRL diagnosis, 262 patients underwent US-FNAB and 4 patients underwent excisional biopsy of the LNs. Pathological characteristics of the patients were categorized retrospectively according to the $8^{\text {th }}$ Edition of the
American Joint Committee on Cancer (AJCC) manual and were reviewed based on histopathological reports by the authors [26]. Among the 112 patients who underwent neoadjuvant chemotherapy before surgery, clinical staging was used instead of pathological staging.

\section{US examinations and US-FNAB procedures}

US examinations were performed by 1 of 5 experienced breast radiologists using SONOLINE Antares (Siemens Medical Systems, Issaquah, WA, USA) or Philips iU22 (Philips Healthcare, Best, The Netherlands) or LOGIC E9 (GE Healthcare, Milwaukee, WI, USA) devices equipped with linear-array transducers (7-13 MHz or 5-12 MHz). Postoperative US screening surveillance was performed every 6 months for 5 years after surgery and annually thereafter, while mammography was performed annually.

The scan range of the postoperative US examinations was as follows: both breasts, including mastectomy or breast-conserving surgery site, bilateral axillae, bilateral internal mammary areas and bilateral supraclavicular areas. US examination of the axillary LNs was performed along the axillary artery from the lower axilla to the junction of the axilla and upper arm; for evaluation of the internal mammary LNs, US examination was performed along both sides of the sternum and internal mammary chains; and, finally, for examination of the supraclavicular fossa, the arms were lowered with the chin lifted and the patient supine. US was performed on the both sides of the neck, from the lower margin of the thyroid cartilage to the level of the clavicle. Approximate scanning time was 5 minutes.

Suspicious features of LRL on US included the following: marked hypoechogenicity; round or irregular shape; eccentric cortical thickening; and replacement of the fatty hilum [13]. US-FNAB was recommended for all suspected LRLs found in the axillae, and/or internal mammary areas and/or supraclavicular areas. US-FNAB was performed in LNs with at least one of the abovementioned suspicious features on US by the same radiologists who evaluated the LNs using US. A 23-gauge needle was used for aspiration using the free-hand technique and each lesion was aspirated at least twice.

\section{Data and statistical analysis}

The final diagnosis of LRL was based on pathological results and clinical follow-up. A true-positive finding was defined as an LN with proven malignancy at the subsequent pathological examination. A false-negative finding was defined as an LN that was negative for malignancy at the pathological examination but exhibited an increase in size on the follow-up within 12 months. The positive predictive value (PPV) for each regional $\mathrm{LN}$ area was assessed. Furthermore, the association between LRL and 
clinicopathological characteristics in patients with suspicious features of LRL on US were also evaluated.

Statistical comparisons were performed using the independent $t$-test for parametric variables or Pearson's chi-squared test for nonparametric variables. Univariate and multivariate logistic regression analyses were used to identify risk factors predictive for LRL of the supraclavicular area. In the nuclear grade, we grouped G1 into lower nuclear grade, G2 and G3 into higher nuclear grade. In the $\mathrm{T}$ stage of the primary cancer, we categorized Tis, T1 and T2 into lower T stage, T3 and T4 into higher $\mathrm{T}$ stage. In the $\mathrm{N}$ stage of the primary cancer, we categorized N0 and N1 into lower N stage, N2 and $\mathrm{N} 3$ into higher $\mathrm{N}$ stage. Also, in the overall stage of the primary cancer, we grouped stage 0 and I (IA,IB) into lower overall stage, stage II (IIA,IIB) and stage III (IIIA,IIIB,IIIC) into higher overall stage. The overall stage of the primary cancer was established based on the $\mathrm{T}$ and $\mathrm{N}$ stages, nuclear grade, and hormonal receptor status. Therefore, multivariate analysis was performed in two models: model 1, containing the overall stage; and model 2, including the $\mathrm{T}$ stage, $\mathrm{N}$ stage and nuclear grade. Statistical analyses were performed using SPSS version 21 (IBM Corporation, Armonk, NY, USA) for Windows (Microsoft Corporation, Redmond, WA, USA), and SAS version 9.2 (SAS Inc, Cary, NC, USA). Differences with $\mathrm{p}<0.05$ were considered to be statistically significant.

\section{Results}

\section{Clinicopathological characteristics}

Patient demographics and clinicopathological characteristics are listed in Table I. Of the 266 patients with suspicious findings for LRL on postoperative US, 68 (25.6\%) patients had a higher N stage and $126(47.4 \%)$ patients had a higher overall stage.

\section{Locations and final diagnosis according to the \\ presence of $L R L$}

Of the 280 suspicious findings for LRL in the 266 patients, $74 \mathrm{LNs}$ in 70 patients were histopathological confirmed as LRL [24 (32.4\%) in the supraclavicular fossa (16 in the ipsilateral supraclavicular fossa and the other 8 in the contralateral supraclavicular fossa), 38 (51.4\%) LNs in the axilla and $12(16.2 \%)$ LNs in the internal mammary area] (Table II, fig 1). Of the 206 LNs reported to be benign according to histological analysis, no LNs were false-negative at follow-up within 12 months on US, breast MRI and low dose chest CT. The PPV for postoperative US screening surveillance for LRL detection was higher for LNs in the internal mammary areas and those in the supraclavicular fossae compared with those in the axillae.
Table I. Patient demographic characteristics of women with suspicious LRL on postoperative US after undergoing breast cancer surgery

\begin{tabular}{|c|c|}
\hline Characteristics & $\begin{array}{l}\text { Total } \\
(\mathrm{N}=266)\end{array}$ \\
\hline Age & $59.2 \pm 9.3$ \\
\hline \multicolumn{2}{|l|}{ Primary cancer site } \\
\hline Unilateral & $253(94.0)$ \\
\hline Bilateral & $13(6.0)$ \\
\hline \multicolumn{2}{|l|}{ Underwent operation method for primary cancer } \\
\hline Breast conserving surgery & $83(31.2)$ \\
\hline Mastectomy & $183(68.8)$ \\
\hline \multicolumn{2}{|l|}{ Histologic type of primary cancer } \\
\hline Ductal carcinoma in situ (DCIS) & $10(3.8)$ \\
\hline Invasive ductal carcinoma & $129(48.5)$ \\
\hline Invasive ductal carcinoma with DCIS & $75(28.2)$ \\
\hline Invasive lobular carcinoma & $16(6.0)$ \\
\hline Mucinous carcinoma & $6(2.3)$ \\
\hline Medullary carcinoma & $3(1.1)$ \\
\hline Papillary carcinoma & $7(2.6)$ \\
\hline Other type cancer & $20(7.5)$ \\
\hline Primary tumor size $(\mathrm{mm})$ & $23.4 \pm 15.4$ \\
\hline \multicolumn{2}{|l|}{ Primary cancer stage } \\
\hline \multicolumn{2}{|l|}{ T stage } \\
\hline Tis & $10(3.7)$ \\
\hline $\mathrm{T} 1$ & $118(44.4)$ \\
\hline $\mathrm{T} 2$ & $110(41.4)$ \\
\hline $\mathrm{T} 3$ & $21(7.9)$ \\
\hline $\mathrm{T} 4$ & $7(2.6)$ \\
\hline \multicolumn{2}{|l|}{$\mathrm{N}$ stage } \\
\hline No & $128(48.1)$ \\
\hline N1 & $70(26.3)$ \\
\hline $\mathrm{N} 2$ & $36(13.6)$ \\
\hline N3 & $32(12.0)$ \\
\hline \multicolumn{2}{|l|}{ Histologic grade } \\
\hline G1 & $70(26.3)$ \\
\hline $\mathrm{G} 2$ & $67(25.2)$ \\
\hline G3 & $129(48.5)$ \\
\hline \multicolumn{2}{|l|}{ Hormonal receptor type } \\
\hline Luminal type cancer (luminal A, luminal B) & $98(36.8)$ \\
\hline HER2-positive cancer & $109(41.0)$ \\
\hline Triple negative cancer & $59(22.2)$ \\
\hline \multicolumn{2}{|l|}{ Overall stage of primary cancer } \\
\hline 0 & $10(3.8)$ \\
\hline $\mathrm{I}(\mathrm{IA}, \mathrm{IB})$ & $130(48.9)$ \\
\hline II (IIA, IIB) & $68(25.5)$ \\
\hline III (IIIA, IIIB, IIIC) & $58(21.8)$ \\
\hline
\end{tabular}

The results are expressed as mean \pm standard deviation or mean (\%). 
Table II. Results of locations of suspicious for LRL and the final diagnosis on the postoperative US after undergoing breast cancer surgery

\begin{tabular}{llll}
\hline Location & Axilla & Internal mammary area & Supraclavicular fossa \\
\hline Number of suspicious for LRL on US (N=280) & $178(63.6)$ & $22(7.8)$ & $80(28.6)$ \\
$\quad$ Malignant $(\mathrm{N}=74)$ & $38(51.4)$ & $12(16.2)$ & $24(32.4)$ \\
$\quad$ Benign (N=206) & $140(68.0)$ & $10(4.8)$ & $56(27.2)$ \\
Positive predictive value (\%) & 21.3 & 54.5 & 28.6 \\
\hline
\end{tabular}

The results are expressed as mean (\%). LRL, locoregional recurrence in the lymph node; US, ultrasound.

\section{Predictive factors of the supraclavicular $L R L$}

Table III summarizes the results of univariate and multivariate analysis for predictive factors for supraclavicular LRL. In the univariate analysis, age $(\mathrm{p}=0.004)$, undergoing breast-conserving surgery $(\mathrm{p}=0.043)$, higher overall stage of the primary cancer $(\mathrm{p}=0.015)$, higher $\mathrm{N}$ stage of the primary cancer $(\mathrm{p}=0.002)$, and higher nuclear grade $(\mathrm{p}=0.01)$ were independently associated with supraclavicular LRL, respectively. Primary breast cancers with nuclear grade G3 were more likely to have LRL than were those with nuclear grade G1 $(\mathrm{p}=0.002)$. However, bilaterality, histological type, higher T stage, and hormonal receptor status of the primary cancer were not significantly associated with supraclavicular LRL ( $\mathrm{p}=0.900$, $\mathrm{p}=0.789, \mathrm{p}=0.216, \mathrm{p}=0.899$, respectively).

The multivariate analysis was performed using two models. In model 1, older patient age (odds ratio [OR] 1.060 [95\% confidence interval (CI) 1.026-1.095]; $\mathrm{p}<0.001$ ), undergoing breast-conserving surgery (OR 2.253 [95\% CI 1.184-4.289]; $\mathrm{p}=0.013$ ), and higher overall stage of the primary cancer (OR 2.361 [95\% CI $1.302-4.282] ; p=0.005$ ) were independent predictive factors for LRL of supraclavicular LNs. In model 2, older patient age (OR 1.059 [95\% CI 1.022-1.098]; $\mathrm{p}=0.001$ ), undergoing breast-conserving surgery (OR 3.209 [95\% CI 1.538-6.692]; $\mathrm{p}=0.002$ ), and higher $\mathrm{N}$ stage of the pri- mary cancer (OR 3.086 [95\% CI 1.479-6.441]; $p=0.003$ ) were independent predictive factors for LRL of supraclavicular LNs. However, the nuclear grade of the primary cancer was not significantly associated with LRL of supraclavicular LNs on multivariate analysis $(\mathrm{p}=0.205)$.

\section{Discussion}

The present study focused on the presence of supraclavicular LRL in patients who underwent breast cancer surgery, which was independently associated with higher $\mathrm{N}$ stage and the higher overall stage of the primary breast cancer. Our results suggested that supraclavicular LRL was approximately 3 times (OR 3.086) more likely to occur in patients with higher $\mathrm{N}$ stages or 2.5 times (OR 2.361) more likely to occur in those with higher overall stages. However, in our univariate model and two multivariate models, higher T stage, nuclear grade and hormonal receptor status did not reach statistical significance in association with supraclavicular LRL in patients who underwent breast cancer surgery.

We found that, if supraclavicular LRL was suspected on routine postoperative US screening surveillance, it was very likely to be identified as LRL after histological examination. In other words, the PPV of the US surveillance for supraclavicular LRL was high. Therefore, we

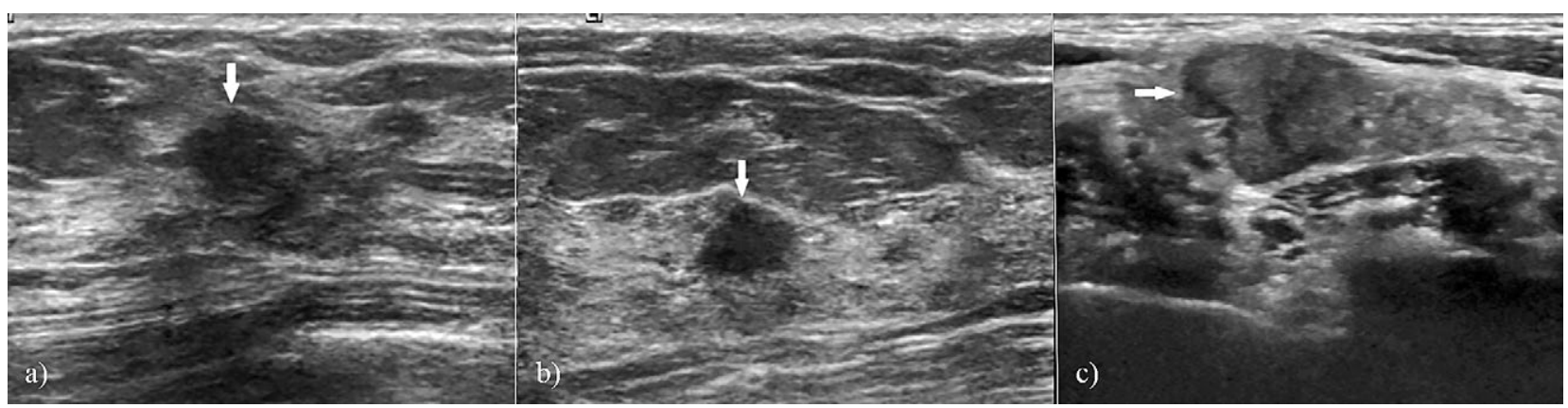

Fig 1. A 57-year-old female patient underwent right breast conserving surgery in 2008: a) and b) a 9 mm-sized irregularly shaped hypoechoic mass was noted at the right 9:00 direction on the preoperative US images. The mass was confirmed as invasive ductal carcinoma and the stage of that was T1bN0 and overall stage IA; c) after 10 years, on the postoperative screening US surveillance, there was an enlarged LN in the right supraclavicular area, which was suspicious for LRL and confirmed as metastatic lymphadenopathy by core needle biopsy. The subtype was same as that of the primary cancer. 
Table III. Univariate and multivariate logistic regression analysis for predicting LRL of supraclavicular lymph nodes

\begin{tabular}{|c|c|c|c|c|c|c|}
\hline & \multicolumn{2}{|l|}{ Univariate analysis } & \multicolumn{2}{|l|}{$\begin{array}{l}\text { Multivariate analysis } \\
\text { model } 1\end{array}$} & \multicolumn{2}{|l|}{$\begin{array}{l}\text { Multivariate analysis } \\
\text { model } 2\end{array}$} \\
\hline & Crude odds ratio & $\mathrm{p}$ value & Adjusted odds ratio & $\mathrm{p}$ value & Adjusted odds ratio & $\mathrm{p}$ value \\
\hline Age (years) & $1.043(1.013-1.074)$ & 0.004 & $1.060(1.026-1.095)$ & $<0.001$ & $1.059(1.022-1.098)$ & 0.001 \\
\hline \multicolumn{7}{|l|}{ Unilateral / bilateral cancer } \\
\hline Unilaterality & Reference & & & & & \\
\hline Bilaterality & $0.926(0.282-3.046)$ & 0.900 & & & & \\
\hline \multicolumn{7}{|l|}{ Surgery } \\
\hline Mastectomy & Reference & & Reference & & Reference & \\
\hline Breast conserving surgery & $1.765(1.017-3.062)$ & 0.043 & $2.253(1.184-4.289)$ & 0.013 & $3.209(1.538-6.692)$ & 0.002 \\
\hline Histologic type & $1.080(0.615-1.897)$ & 0.789 & & & & \\
\hline \multicolumn{7}{|l|}{ Overall stage } \\
\hline Stage 0 and $\mathrm{I}(\mathrm{IA}, \mathrm{IB})$ & Reference & & & & & \\
\hline $\begin{array}{l}\text { Stage II (IIA, IIB) and } \\
\text { III (IIIA, IIIB, IIIC) }\end{array}$ & $2.011(1.146-3.528)$ & 0.015 & $2.361(1.302-4.282)$ & 0.005 & & \\
\hline \multicolumn{7}{|l|}{ T stage } \\
\hline Tis, $\mathrm{T} 1$, and $\mathrm{T} 2$ & Reference & & & & & \\
\hline $\mathrm{T} 3$ and $\mathrm{T} 4$ & $0.488(0.157-1.521)$ & 0.216 & & & & \\
\hline \multicolumn{7}{|l|}{$\mathrm{N}$ stage } \\
\hline N0 and N1 & Reference & & & & Reference & \\
\hline $\mathrm{N} 2$ and N3 & $2.660(1.415-5.000)$ & 0.002 & & & $3.086(1.479-6.441)$ & 0.003 \\
\hline Nuclear grade & & 0.010 & & & & 0.205 \\
\hline G1 & Reference & & & & Reference & \\
\hline G2 & $2.632(1.065-6.502)$ & 0.036 & & & $1.348(0.498-3.652)$ & 0.557 \\
\hline G3 & $3.483(1.553-7.811)$ & 0.002 & & & $2.097(0.861-5.110)$ & 0.103 \\
\hline Hormonal receptor status & & 0.899 & & & & \\
\hline Luminal type & Reference & & & & & \\
\hline HER2-positive type & $1.020(0.537-1.936)$ & 0.952 & & & & \\
\hline Triple-negative type & $1.183(0.555-2.522)$ & 0.663 & & & & \\
\hline
\end{tabular}

found that US was a reliable modality for the evaluation of the supraclavicular LN. Moreover, we could identify whether patients had a high potential for supraclavicular LRL using staging of the primary breast cancer. In cases for which the high-stage primary breast cancer is confirmed on the imaging evaluation before neoadjuvant chemotherapy or on the final surgical pathology, postoperative US screening surveillance for the supraclavicular areas must be carefully realized. Furthermore, we may expect that postoperative routine US scanning of the supraclavicular area would improve patient prognosis by enabling early detection of supraclavicular LRL.

Many previous studies have reported that younger age is a significant risk factor for locoregional recurrence of breast cancer [27-32]. Unlike previous studies investigating age, we demonstrated that older age was also independently associated with supraclavicular LRL. While most previous studies compared young age and old age as subgroups, we set the age as a continuous variable. In addition, the mean age of our study population was somewhat higher than in other studies [32]. In our study group, older patients tended to have a higher stage of primary cancer and that could explain the association between the older age and the supraclavicular LRL. In addition, there was also an independent association between supraclavicular LRL and previous breastconserving surgery, unlike other studies [33,34]. Patients undergoing mastectomy are more likely to have a higher stage of primary cancer than are those undergoing breastconserving surgery and this may be interpreted as a result of the association between supraclavicular LRL and higher staging of the primary breast cancer. The precise association between supraclavicular LRL and age and breast-conserving surgery should be determined through further larger-scale studies.

There were several limitations to our study. First, the retrospective design of the study. We excluded patients who underwent postoperative screening surveillance 
using CT or MRI without US and those with palpable lesion(s) in the locoregional areas; thus, selection bias was inevitable. Second, patients who underwent neoadjuvant chemotherapy underwent clinical staging instead of pathological staging. The exact pathological staging was unknown; therefore, this may have affected the outcome. Third, a small number of cases with LRL ( $\mathrm{n}=74)$, especially with supraclavicular LRL $(\mathrm{n}=24)$, was analyzed. Fourth, we did not evaluate interobserver variability in the US interpretation for LRL. Fifth, we focused only on supraclavicular LRL not internal mammary LRL. However, the incidence of internal mammary LRL was relatively lower than that of axillary or supraclavicular LRL in a previous study [35] and also in our study. Therefore, the effect of excluding internal mammary LRL on the results of our study is believed to be minimal. Finally, clinicopathological factors were compared between true positive - histologic confirmed LRL - and false positive - histologic confirmed negative for malignancy - groups. The false positive group did not represent the non-LRL group.

In conclusion, in patients who underwent breast cancer surgery, the higher overall stage of primary breast cancer and the higher $\mathrm{N}$ stage of primary breast cancer are predictive factors of supraclavicular LRL. And routine US supraclavicular scanning is useful for the detection of supraclavicular LRL in breast cancer patients with a higher stage.

Acknowledgement: This work was supported by the Dong-A University research fund.

\section{Conflict of interest: none}

\section{References}

1. Bonotto M, Gerratana L, Poletto E, et al. Measures of outcome in metastatic breast cancer: insights from a real-world scenario. Oncologist 2014;19:608-615.

2. Forouzanfar MH, Foreman KJ, Delossantos AM, et al. Breast and cervical cancer in 187 countries between 1980 and 2010: a systematic analysis. Lancet 2011;378:14611484.

3. Berry DA, Cronin KA, Plevritis SK, et al. Effect of Screening and Adjuvant Therapy on Mortality from Breast Cancer. N Engl J Med 2005;353:1784-1792.

4. Lu WL, Jansen L, Post WJ, Bonnema J, Van de Velde JC, De Bock GH. Impact on survival of early detection of isolated breast recurrences after the primary treatment for breast cancer: a meta-analysis. Breast Cancer Res Treat 2009; 114:403-412.

5. Favourable and unfavourable effects on long-term survival of radiotherapy for early breast cancer: an overview of the randomised trials. Early Breast Cancer Trialists' Collaborative Group. Lancet 2000;355:1757-1770.
6. Clemons M, Danson S, Hamilton T, Goss P. Locoregionally recurrent breast cancer: incidence, risk factors and survival. Cancer Treat Rev 2001;27:67-82.

7. Wapnir IL, Anderson SJ, Mamounas EP, et al. Prognosis after ipsilateral breast tumor recurrence and locoregional recurrences in five National Surgical Adjuvant Breast and Bowel Project node-positive adjuvant breast cancer trials. J Clin Oncol 2006;24:2028-2037.

8. Wapnir IL, Aebi S, Geyer CE, et al. A randomized clinical trial of adjuvant chemotherapy for radically resected locoregional relapse of breast cancer: IBCSG 27-02, BIG 1-02, and NSABP B-37. Clin Breast Cancer 2008;8:287292.

9. Anderson SJ, Wapnir I, Dignam JJ, et al. Prognosis after ipsilateral breast tumor recurrence and locoregional recurrences in patients treated by breast-conserving therapy in five National Surgical Adjuvant Breast and Bowel Project protocols of node-negative breast cancer. J Clin Oncol 2009;27:2466-2473.

10. Nagashima T, Sakakibara M, Kadowaki M, et al. Response rate to neoadjuvant chemotherapy measured on imaging predicts early recurrence and death in breast cancer patients with lymph node involvements. Acta Radiol 2011;52:241246.

11. Monticciolo DL, Newell MS, Moy L, Niell B, Monsees B, Sickles EA. Breast Cancer Screening in Women at HigherThan-Average Risk: Recommendations From the ACR. J Am Coll Radiol 2018;15:408-414.

12. D’Orsi CJ, Sickles EA, Medelson EB, et al. ACR BIRADS ${ }^{\circledR}$ Atlas, Breast Imaging Reporting and Data System. Reston, VA; American College of Radiology, 2013.

13. Moon HJ, Kim MJ, Kim EK, et al. US surveillance of regional lymph node recurrence after breast cancer surgery. Radiology 2009;252:673-681.

14. Suh YJ, Kim MJ, Kim EK, Moon HJ, Kim SI, Park BW. Value of ultrasound for postoperative surveillance of asian patients with history of breast cancer surgery: a single-center study. Ann Surg Oncol 2013;20:3461-3468.

15. Kim HJ, Kwak JY, Choi JW, et al. Impact of US surveillance on detection of clinically occult locoregional recurrence after mastectomy for breast cancer. Ann Surg Oncol 2010;17:2670-2676.

16. Balu-Maestro C, Bruneton JN, Geoffray A, Chauvel C, Rogopoulos A, Bittman O. Ultrasonographic posttreatment follow-up of breast cancer patients. J Ultrasound Med 1991;10:1-7.

17. Lee JH, Kim EK, Oh JY, et al. US screening for detection of nonpalpable locoregional recurrence after mastectomy. Eur J Radiol 2013;82:485-489.

18. Liu YQ, Dong JN, Meng QX, Sun P, Zhang J. Ultrasound for postoperative surveillance after mastectomy in patients with breast cancer: A retrospective study. Medicine (Baltimore) 2017;96:e9244.

19. van Tienhoven G, Voogd AC, Peterse JL, et al. Prognosis after treatment for loco-regional recurrence after mastectomy or breast conserving therapy in two randomised trials (EORTC 10801 and DBCG-82TM). EORTC Breast Cancer 
Cooperative Group and the Danish Breast Cancer Cooperative Group. Eur J Cancer 1999;35:32-38.

20. Abi-Raad R, Boutrus R, Wang R, et al. Patterns and risk factors of locoregional recurrence in T1-T2 node negative breast cancer patients treated with mastectomy: implications for postmastectomy radiotherapy. Int $\mathrm{J}$ Radiat Oncol Biol Phys 2011;81:e151-e157.

21. Willner J, Kiricuta I, Kolbl O, Bohndorf W. Supraclavicular lymph-node recurrence of breast-cancer. Oncol Rep 1994;1:1235-1245.

22. Schmoor C, Sauerbrei W, Bastert G, Schumacher M. Role of isolated locoregional recurrence of breast cancer: results of four prospective studies. J Clin Oncol 2000;18:16961708.

23. Harris EE, Hwang WT, Seyednejad F, Solin LJ. Prognosis after regional lymph node recurrence in patients with stage I-II breast carcinoma treated with breast conservation therapy. Cancer 2003;98:2144-2151.

24. Houssami N, Ciatto S, Martinelli F, Bonardi R, Duffy SW. Early detection of second breast cancers improves prognosis in breast cancer survivors. Ann Oncol 2009;20:15051510 .

25. Pedersen AN, Moller S, Steffensen KD, et al. Supraclavicular recurrence after early breast cancer: a curable condition? Breast Cancer Res Treat 2011;125:815-822.

26. Hortobagyi GN, Connolly JL, D’Orsi CJ, et al. AJCC Cancer Staging Manual, Eight Edition, 2017.

27. Clarke DH, Lê MG, Sarrazin D, et al. Analysis of localregional relapses in patients with early breast cancers treated by excision and radiotherapy: experience of the Institut Gustave-Roussy. Int J Radiat Oncol Biol Phys 1985;11:137-145.
28. Recht A, Connolly JL, Schnitt SJ, et al. The effect of young age on tumor recurrence in the treated breast after conservative surgery and radiotherapy. Int J RadiatOncol Biol Phys1988;14:3-10.

29. Cao JQ, Olson RA, Tyldesley SK. Comparison of recurrence and survival rates after breast-conserving therapy and mastectomy in young women with breast cancer. Curr Oncol 2013;20:e593-e601.

30. Brandt J, Garne JP, Tengrup I, Manjer J. Age at diagnosis in relation to survival following breast cancer: a cohort study. World J Surg Oncol 2015;13:33.

31. He XM, Zou DH. The association of young age with local recurrence in women with early-stage breast cancer after breast-conserving therapy: a meta-analysis. Sci Rep 2017;7:11058.

32. Choi Y, Hwang TS, Jeong AR, et al. Clinicopathological markers associated with recurrence in ductal carcinoma in situ of breast by age group. Korean J Clin Oncol 2018;14:15-20.

33. Zhou X, Li Y. Local Recurrence after Breast-Conserving Surgery and Mastectomy Following Neoadjuvant Chemotherapy for Locally Advanced Breast Cancer - a MetaAnalysis. Breast Care (Basel) 2016;11:345-351.

34. Sun Y, Liao M, He L, Zhu C. Comparison of breast-conserving surgery with mastectomy in locally advanced breast cancer after good response to neoadjuvant chemotherapy: A PRISMA-compliant systematic review and meta-analysis. Medicine 2017;96:e8367.

35. Livi L, Paiar F, Simontacchi G, et al. Loco regional failure pattern after lumpectomy and breast irradiation in 4,185 patients with T1 and T2 breast cancer. Implications for nodal irradiation. Acta Oncol 2006;45:564-570. 